\title{
Correspondence
}

Arch. Dis. Childh., 1969, 44, 285.

\section{Hazards of Enuresis Alarms}

Sirs,

Enuresis blankets with buzzer alarms of a kind widely prescribed for bed-wetting children may occasionally cause ulceration and scarring.

Case 1. A boy of 8 years presented with lesions on the thighs and buttocks which had begun to appear 2 months previously. There was no irritation or discomfort of any kind. Each began as a red spot which later ulcerated and then finally scarred. Examination revealed 26 scars, nearly all of which were on the right thigh or right buttock. Each scar was circular, 2-10 $\mathrm{mm}$. diameter, and depressed in the centre.

Case 2. A boy of 7 years, the brother of Case 1, presented with almost identical lesions consisting of 10 scars on the right thigh and right buttock which had appeared during the previous 2 months. The scars were preceded by the same changes as in Case 1 . They were circular, 2-8 $\mathrm{mm}$. diameter, and had not been noticed by the patient till his attention was drawn to them.

Case 3. A girl of 9 years was found to have a single depressed circular scar about $5 \mathrm{~mm}$. diameter on the right thigh. This lesion had been noted by the patient just after the acquisition of an enuresis alarm, which was discontinued after a few nights' use.

Cases 1 and 2 used identical models (Fig.), consisting of two metal foil electrodes $41 \times 71 \mathrm{~cm}$. $(16 \times 28 \mathrm{in}$.$) , the upper of which was perforated$ by holes $1.9 \mathrm{~cm}$. (3 in.) diameter and $3.8 \mathrm{~cm}$. ( $1 \frac{1}{2}$ in.) apart. The electrodes connected with a box containing a 6-volt dry battery. A switch selected the alarm, which was either a buzzer or a small lamp. The circuit was completed when the patient wet the bed, moistening the sheet separating the two electrodes and permitting a current to pass between them. Either the buzzer or the lamp was activated, depending on the position of the switch. The third patient used a 4-volt model which had only a buzzer and no lamp, but was otherwise similar.

The following facts indicate that the scars in Cases 1 and 2 were due to prolonged contact of the patient's urine-soaked skin with the 'live' metal foil electrodes. The scars were limited to the skin in closest apposition to the enuresis blanket, being localized to the side on which the patient habitually slept (the right side in each case). Nearly all the scars were below the waist. The shape and size of the scars corresponded with the perforations on the upper of the two foil electrodes. In some areas the pattern of the scars corresponded spatially with the perforations. Each patient stated that he frequently switched his buzzer off in order to spare his brother the inconvenience of being awakened by it. Thus he was not woken by bed wetting though the lamp glowed, and the current was able to pass continuously through those parts of the skin corresponding to the perforations of the upper foil electrode.

In Case 3 the battery of the apparatus had been allowed to run down and when the patient urinated the buzzer sounded too feebly to waken her, thus creating a situation identical to that of Cases 1 and 2 .

P. Borrie and J. C. B. Fenton (Brit. med. J., $1966,2,151)$ reported two cases of ulceration caused by enuresis blankets, and attributed the lesions in each instance to failure of the alarm due to a run-down battery. They emphasized the painless nature of the lesions, which was a feature of the patients reported here.

In all three of our cases the cause of the skin injuries was faulty design of the apparatus resulting

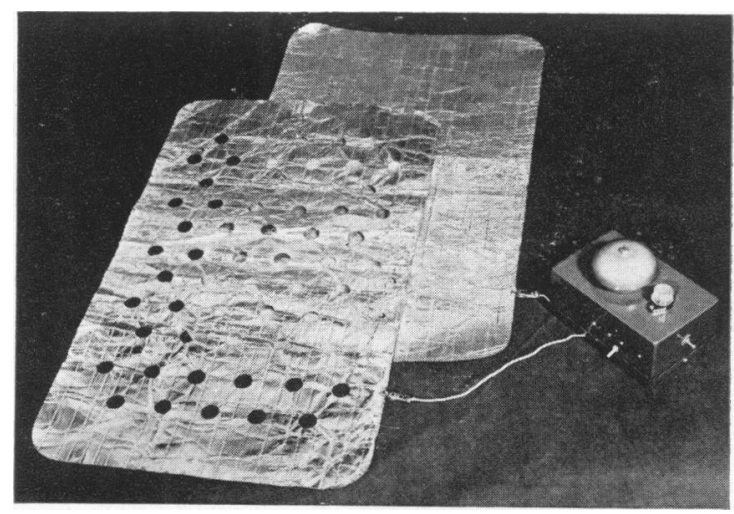

FIg.-Enuresis alarm unit as used by Cases 1 and 2. 
in failure of the alarm to wake the patient despite flow of current due to bedwetting. Existing apparatus should therefore contain the following modifications. (1) Perforations in the upper foil electrode of a reduced diameter: these, while permitting seepage of urine and immediate completion of the electrical circuit, would prevent contact of skin with the lower electrode. (2) An alarm system which cannot be put out of action by the user, except by switching off the whole electrical system.

M. W. Greaves

University Department of Dermatology,

Newcastle upon Tyne 1.

The following articles will appear in future issues of this Journal:

Total Lipodystrophy. By Angela Fairney, George Lewis, and Dennis Cottom.

Treatment of Homocystinuria with Pyridoxine. A Preliminary Study. By N. A. J. Carson and I. J. Carré.

Plasmo Amino Acid Disturbance in Infancy. I. Hypermethioninaemia and Transient Tyrosinaemia. By G. M. Komrower and A. J. Robins.

Autonomic Nerves in Rectum and Colon in Hirschsprung's Disease. By J. R. Garrett, E. R. Howard, and H. H. Nixon.

Significance of Hypocalcaєmia in Neonatal Convulsions. By J. H. Keen.

Temporary Loss of a Metabolic Response to Cold Stress in Infants of Low Birthweight. By E. N. Hey and G. Katz. Use of THAM and Sodium Bicarbonate in Correcting Acidosis in Asphyxiated Newborns. By D. Berg, M. Mülling, and E. Saling.

Variations in the Pattern of Pubertal Changes in Girls. By W. A. Marshall and J. M. Tanner.

Screening of Pyruvate Kinase Deficiency and Glucose-6-phosphate Dehydrogenase Deficiency in the Chinese Newborn in Hong Kong. By Robert H. P. Fung, Yeung Kwok Keung, and Gabriel S. H. Chung.

Measurement of Response to Isoprenaline in Asthmatic Children. By Elizabeth A. Featherby, Tzong-Ruey Weng, and Henry Levison.

Ventilatory Function in Infants with Cystic Fibrosis. Physiological Assessment of Inhalation Therapy. By P. D. Phelan, M. Gracey, H. E. Williams, and Charlotte M. Anderson.

The Treatment of Hydrocephalus by the Use of Saphenous Vein Graft. By T. E. Udwadia.

Blood Glucose and Serum Insulin Levels in Children with Cystic Fibrosis. By A. D. Milner.

Wolman's Disease. A Rare Lipidosis with Adrenal Calcification. By W. C. Marshall, B. G. Ockenden, A. S. Fosbrooke, and J. M. Cumings.

Studies on Hypoglycaemia in Small-for-dates Newborns. By D. Blum, J. Dodion, H. Loeb, P. Wilkin, and P. O. Hubinont.

Central Pontine Myelinolysis in Childhood and Adolescence. By Thomas E. Cadman and Lucy Balian Rorke. Ectopic Glial Tissue in the Occipital Scalp. By Frederick E. Jackson and B. S. Moore.

Physio-pathology of the Internal Anal Sphincter. By Bernard Duhamel.

The Captive Mother. By S. R. Meadow.

A Screening Method for Liver Glycogen Disease. By J. Fernandes, F. Huijing, and J. E. van de Kamer. 